\title{
STOKES FLOW \\ IN A TWO-DIMENSIONAL SPATIALLY PERIODIC BOUNDARY DRIVEN BY A COMBINATION OF LINE SINGULARITIES
}

\author{
BY \\ K. MA AND D. W. PRAVICA \\ University of Toronto, Toronto, Ontario, Canada
}

\begin{abstract}
The analytical solution is obtained for two-dimensional creeping flow generated by a source-sink combination located on a spatially periodic boundary. Separation is found to occur when the source and sink are located on concave regions of the boundary. The velocity profile of the two-dimensional Poiseuille flow for a channel is compared to that of the circle problem and the rough channel obtained from the analytical solution. It is found that the mid-channel velocity (or pressure gradient) of the rough case is greater (less) than that of the circle problem.
\end{abstract}

§1. Introduction. The study of viscous flow through a corrugated pipe has received considerable interest in the fields of meteorology and chemical engineering due to its usage to model flow over rough topography and in porous media respectively. The reader is referred to [1-3] for literature in these areas.

This paper considers the two-dimensional Stokes flow generated by a source and sink which are located on opposite sides of a bounded corrugated tube. The boundary of the conformally mapped figure consists of $n \geq 1$ corrugations which can be made arbitrarily sharp. The positions of these peaks and their geometry dramatically effect the fluid flow. In many cases, there is boundary separation as well as the appearance of separatrices.

The analytical solution to the above Stokes flow problem is used to approximate a Poiseuille flow through a rough channel since such Poiseuille flows are used to model laminar pipe flows found in many chemical-industrial, hydraulic, and biophysical problems. The velocity profile of this rough channel flow is compared to that of a smooth channel obtained from the same analytical solution. It is found that the corrugated boundary of the rough channel results in a greater mid-channel velocity than the smooth channel which has smooth boundary. The variation of the velocity profile, however, is stable with respect to surface roughness and this is in agreement with the assumptions made in lubrication theory [ $4 \mathrm{j}$. For more literature on the stability of such flow with the addition of inertial effects (nonzero Reynolds number) see [5].

Received January 28, 1991 and, in revised form, March 28, 1991.

(C) 1992 Brown University 
$\S 2$. Boundary geometry. The mapping considered here will be the fluted column transformation (see [7] and [8])

$$
z=x+i y=r e^{i \theta}=\zeta+\varepsilon \zeta^{n+1}
$$

for $n \geq 1$, where

$$
\zeta=\rho e^{i \phi}, \quad 0 \leq \rho \leq 1, \quad-\pi<\phi \leq \pi .
$$

It is conformal in $|\zeta| \leq 1$ for $|\varepsilon|(n+1)<1$ and maps $|\zeta| \leq 1$ from the $\zeta$-plane to a compact region with a corrugated boundary of $n$ peaks between $r=1 \pm \varepsilon$ in the $z$-plane. (2.1) implies

$$
x=\rho \cos \phi+\varepsilon \rho^{n+1} \cos (n+1) \phi, \quad y=\rho \sin \phi+\varepsilon \rho^{n+1} \sin (n+1) \phi .
$$

Two different ranges of $\varepsilon$ are considered here, namely

$$
\varepsilon \in I_{1}=[0,1 /(n+1)) \quad \text { or } \quad \varepsilon \in I_{2}=(-1 /(n+1), 0] .
$$

It can be shown that the boundary $(\rho=1)$ is always smooth, but as $|\varepsilon| \rightarrow 1 /(n+1)$ there are points that appear which approach the shape of a cusp pointing towards the center. In particular the boundary is convex for $|\varepsilon| \leq 1 /(n+1)^{2}$; however for $1 /(n+1)^{2}<|\varepsilon|<1 /(n+1)$ smooth concavities appear. It is easily shown that the curvature at the peak of a cusp is

$$
\kappa=\frac{|1-| \varepsilon\left|(n+1)^{2}\right|}{(1-|\varepsilon|(n+1))^{2}} .
$$

For odd $n(\varepsilon>0, \varepsilon \rightarrow 1 /(n+1))$ one cusp appears on the $x$-axis. For even $n$ there is either no cusp on the $x$-axis $(\varepsilon>0)$, or a cusp at the points of intersection of the boundary with the $x$-axis $(\varepsilon<0, \varepsilon \rightarrow-1 /(n+1))$. So three cases are considered for $(2.1)$;

(i) odd $n, \varepsilon \in I_{1}$,

(ii) even $n, \varepsilon \in I_{1}$,

(iii) even $n, \varepsilon \in I_{2}$.

$\S 3$. The flow problem and method of solution. For steady two-dimensional Stokes flow, the governing Stokes equations in nondimensional form are

$$
\begin{gathered}
\nabla_{1}^{2} \underline{q}=\nabla p, \\
\nabla \cdot \underline{q}=0,
\end{gathered}
$$

where $q$ is the velocity field, and $p$ is the pressure field of the flow respectively. A Stokes stream function $\psi=\psi(x, y)$ is introduced as

$$
\underline{q}=\operatorname{curl}(\psi \hat{k}),
$$

where $\hat{k}$ is a unit vector perpendicular to the plane of motion, so that $(3.1 \mathrm{~b})$ is automatically satisfied. Substituting (3.2) into (3.1a) and taking the curl of both 
sides or eliminating the pressure field gives

$$
\nabla_{1}^{4} \psi=0
$$

where

$$
\nabla_{1}^{2}=\frac{\partial^{2}}{\partial x^{2}}+\frac{\partial^{2}}{\partial y^{2}}, \quad \nabla_{1}^{4}=\left(\nabla_{1}^{2}\right)^{2}
$$

Using the fluted column transformation, the appropriate boundary conditions for a line source and sink located on the boundary intersecting the $x$-axis are

$$
\psi(1, \phi)=M \operatorname{sgn} \phi, \quad \psi_{\rho}(1, \phi)=0, \quad-\pi<\phi \leq \pi,
$$

which are the no-seepage (except at source and sink) and no-slip boundary conditions of the problem respectively.

To solve $(3.3 \mathrm{a}, \mathrm{b})$, set $\psi=\psi_{0}+\psi_{1}$ such that

$$
\nabla_{\rho}^{2} \psi_{0}=0: \quad \psi_{0}(1, \phi)=M \operatorname{sgn} \phi, \quad \nabla_{\rho}^{2}=\frac{\partial^{2}}{\partial \rho^{2}}+\frac{1}{\rho} \frac{\partial}{\partial \rho}+\frac{1}{\rho^{2}} \frac{\partial^{2}}{\partial \phi^{2}} .
$$

Note that the solution of the Laplace equation is invariant under a conformal mapping, i.e., $\nabla_{1}^{2} \psi_{0}=0$, and hence $\nabla_{1}^{4} \psi_{0}=0$. Then the corresponding boundary value problem for $\psi_{1}$ is

$$
\left.\begin{array}{r}
\nabla_{1}^{4} \psi_{1}=0: \quad \psi_{1}(1, \phi)=0 \\
\psi_{1 \rho}(1, \phi)=-\psi_{0 \rho}(1, \phi)
\end{array}\right\} \quad-\pi<\phi \leq \pi .
$$

The solution of (3.4), easily obtained by standard techniques, is

$$
\psi_{0}=\frac{2 M}{\pi} \sum_{m=1}^{\infty}\left(\frac{1-(-1)^{m}}{m}\right) \rho^{m} \sin (m \phi)=\frac{2 M}{\pi} \tan ^{-1}\left[\frac{2 \rho \sin \phi}{1-\rho^{2}}\right] .
$$

Now a suitable solution of (3.5) can be chosen, by using the fact that $\psi(1, \phi)$ is an odd function. The transformation (2.1) suggests that we assume a solution of the form

$$
\begin{aligned}
\psi_{1} & =\phi_{0}+x \phi_{1}-y \phi_{2}, \\
\nabla_{1}^{2} \phi_{j} & =0, \quad j=0,1,2, \\
\phi_{0} & =\sum_{m=1}^{+\infty} A_{m} \rho^{m} \sin (m \phi), \\
\phi_{1} & =\sum_{m=1}^{+\infty} B_{m} \rho^{m+1} \sin (m+1) \phi, \\
\phi_{2} & =\sum_{m=1}^{+\infty} B_{m} \rho^{m+1} \cos (m+1) \phi .
\end{aligned}
$$


Applying the boundary conditions for $\psi_{1}$ from (3.5), it can be shown that the $A_{m}$ 's and $B_{m}$ 's satisfy (after some algebra)

$$
\begin{gathered}
A_{m}= \begin{cases}-B_{m}-\varepsilon\left(B_{n+m}-B_{n-m}\right), & 1 \leq m \leq n-1, \\
-B_{m}-\varepsilon B_{n+m}, & n \leq m,\end{cases} \\
B_{m}+\varepsilon\left[(n+1) B_{n+m}-(n+1-m) B_{n-m}\right]=\frac{M}{\pi}\left((-1)^{m}-1\right), \quad 1 \leq m \leq n-1, \\
B_{m}+\varepsilon(n+1) B_{m+n}=\frac{M}{\pi}\left((-1)^{m}-1\right), \quad n \leq m .
\end{gathered}
$$

The solution for the $B_{m}$ 's are (see Appendix (A.2) and (A.4))

$$
B_{m}= \begin{cases}\frac{M}{\pi}\left[\frac{\left((-1)^{m}-1\right)+\Omega_{m}}{1-\varepsilon^{2}(n+1)^{2}}\right], & 1 \leq m \leq n-1, \\ \frac{M}{\pi}\left[\frac{\left((-1)^{m}-1\right)-\varepsilon(n+1)\left((-1)^{m+n}-1\right)}{1-\varepsilon^{2}(n+1)^{2}}\right], & n \leq m,\end{cases}
$$

where

$$
\begin{aligned}
& \Omega_{m}=-\varepsilon\left[\frac{\left((-1)^{m+n}-1\right) m+\varepsilon\left((-1)^{m}-1\right)(n-m)(n+1-m)}{1-\varepsilon^{2}(m+1)(n+1-m)}\right], \\
& \Omega_{m}=0, \quad n=1, \quad m=0,1,
\end{aligned}
$$

and the $\Omega_{m}$ 's satisfy the identity

$$
\Omega_{m}-\varepsilon(n+1-m) \Omega_{n-m}=\varepsilon m\left(1-(-1)^{m+n}\right), \quad 1 \leq m \leq n-1 .
$$

Substitute (3.7b) and (3.8a,b) into (3.7a) and sum the required series for $\psi_{1}$ by standard techniques. After some manipulations

$$
\begin{aligned}
\psi_{1}= & \frac{M}{\pi\left(1-\varepsilon^{2}(n+1)^{2}\right)} \\
& \times\left\{2 \rho \left[\frac{\left[\left(1-\rho^{2}\right)-\varepsilon^{2}(n+1)\left(1-\rho^{2 n+2}\right)\right]\left(1+\rho^{2}\right) \sin \phi}{1-2 \rho^{2} \cos 2 \phi+\rho^{4}}\right.\right. \\
+\frac{\varepsilon \rho^{n}\left(\left(1-\rho^{4}\right)-(n+1)\left(1-\rho^{2}\right)\right) \sin (n+1) \phi}{1-2 \rho^{2} \cos 2 \phi+\rho^{4}} & \left.+\frac{\varepsilon \rho^{n}(n+1) \rho^{2}\left(1-\rho^{2}\right) \sin (n-1) \phi}{1-2 \rho^{2} \cos 2 \phi+\rho^{4}}\right] \\
& \left.+\sum_{m=1}^{n-1}\left[\Omega_{m}\left(\rho^{m+2}-\rho^{m}\right)-\varepsilon \Omega_{n-m}\left(\rho^{2 n+2-m}-\rho^{m}\right)\right] \sin m \phi\right\} .
\end{aligned}
$$


Adding (3.6) and (3.9) gives the solution of (3.3a,b),

$$
\begin{aligned}
& \psi=\psi_{0}+\psi_{1} \\
& =\frac{M}{\pi}\left\{2 \tan ^{-1}\left[\frac{2 \rho \sin \phi}{1-\rho^{2}}\right]+\frac{1}{1-\varepsilon^{2}(n+1)^{2}}\right. \\
& \quad \times\left[\sum_{m=1}^{n-1}\left(\Omega_{m}\left(\rho^{m+2}-\rho^{m}\right)-\varepsilon \Omega_{n-m}\left(\rho^{2 n+2-m}-\rho^{m}\right) \sin m \phi\right]\right. \\
& +\frac{2 \rho}{\left(1-\varepsilon^{2}(n+1)^{2}\right)}\left[\frac{\left[\left(1-\rho^{2}\right)-\varepsilon^{2}(n+1)\left(1-\rho^{2 n+2}\right)\right]\left(1+\rho^{2}\right) \sin \phi}{1-2 \rho^{2} \cos 2 \phi+\rho^{4}}\right. \\
& +\frac{\varepsilon \rho^{n}\left(\left(1-\rho^{4}\right)-(n+1)\left(1-\rho^{2}\right)\right) \sin (n+1) \phi}{1-2 \rho^{2} \cos 2 \phi+\rho^{4}} \\
& \left.\left.+\frac{\varepsilon \rho^{n}(n+1) \rho^{2}\left(1-\rho^{2}\right) \sin (n-1) \phi}{1-2 \rho^{2} \cos 2 \phi+\rho^{4}}\right]\right\} .
\end{aligned}
$$

From this the vorticity can be found as

$$
\begin{aligned}
\omega(\rho, \phi ; \varepsilon) & =\nabla_{1}^{2} \psi \\
& =\nabla_{1}^{2} \psi_{1} \\
& =\left|\frac{d z}{d \zeta}\right|^{-2} \nabla_{\rho}^{2} \psi_{1} \\
& =\frac{\nabla_{\rho}^{2} \psi_{1}}{1+2 \varepsilon(n+1) \rho^{n} \cos n \phi+\varepsilon^{2}(n+1)^{2} \rho^{2 n}},
\end{aligned}
$$

where $\nabla_{\rho}^{2}$ is defined in (3.4), and thus

$$
\begin{aligned}
W(\rho, \phi) & \equiv \nabla_{\rho}^{2} \psi_{1} \\
& =2\left[\left(\frac{\partial x}{\partial \rho} \frac{\partial \phi_{1}}{\partial \rho}+\frac{1}{\rho^{2}} \frac{\partial x}{\partial \phi} \frac{\partial \phi_{1}}{\partial \phi}\right)-\left(\frac{\partial y}{\partial \rho} \frac{\partial \phi_{2}}{\partial \rho}+\frac{1}{\rho^{2}} \frac{\partial y}{\partial \phi} \frac{\partial \phi_{2}}{\partial \phi}\right)\right] .
\end{aligned}
$$


Straightforward differentiation gives for $(3.11 \mathrm{~b})$

$$
\begin{aligned}
& W(\rho, \phi)=\frac{4 M}{\pi\left(1-\varepsilon^{2}(n+1)^{2}\right)} \\
& \times\left\{\sum_{m=1}^{n-1}\left[(m+1) \Omega_{m} \rho^{m}-\varepsilon(n+1)(n+1-m) \Omega_{n-m} \rho^{2 n-m}\right] \sin m \phi\right. \\
& +\frac{1}{\left(1-2 \rho^{2} \cos 2 \phi+\rho^{4}\right)^{2}} \\
& \times\left[\left[-4\left(1-\varepsilon^{2}(n+1)^{2}\right)\left(1+3 \rho^{2}\right)+2 \varepsilon^{2}(n+1)^{2} n \rho^{2 n}\left(1-\rho^{4}\right)\right)\right] \\
& \times \rho\left(1-\rho^{2}\right) \sin \phi+2 \varepsilon(n+1) \rho^{n+1}\left(1-\rho^{2}\right)^{2} \\
& \times\left((n+2) \sin (n+1) \phi-\left(n \rho^{2}-2\right) \sin (n-1) \phi\right) \\
& +4 \rho^{2} \sin ^{2} \phi\left\{\left[-4 \rho^{2}+\varepsilon^{2}(n+1)^{2} \rho^{2 n}\left(4 \rho^{2}+2 n\left(1+\rho^{2}\right)\right)\right] \rho \sin \phi\right. \\
& +2 \varepsilon(n+1) \rho^{n+1}\left[\left(n-2 \rho^{2}\right) \sin (n+1) \phi\right. \\
& \left.\left.\left.\left.-\rho^{2}(n+2) \sin (n-1) \phi\right]\right\}\right]\right\} \text {. }
\end{aligned}
$$

Combining (3.11a) and $(3.11 \mathrm{c})$ gives $\omega(\rho, \phi ; \varepsilon)$ in general. The boundary vorticity is, in particular,

$$
\begin{aligned}
& \omega(1, \phi ; \varepsilon)=\frac{4 M}{\pi\left(1-\varepsilon^{2}(n+1)^{2}\right)\left(1+2 \varepsilon(n+1) \cos n \phi+\varepsilon^{2}(n+1)^{2}\right)} \\
& \times\left\{\sum_{m=1}^{n-1}\left[(m+1) \Omega_{m}-\varepsilon(n+1)(n+1-m) \Omega_{n-m}\right] \sin m \phi\right. \\
& \left.\quad+\frac{-2\left(1-\varepsilon^{2}(n+1)^{3}\right) \sin \phi+\varepsilon(n+1)((n-2) \sin (n+\phi-(n+2) \sin (n-1) \phi)}{2 \sin ^{2} \phi}\right\} .
\end{aligned}
$$

Separation near the boundary can be detected by determining the zeros of $\omega(1, \phi ; \varepsilon)$ or of the bracket on the right-hand side of (3.12). The fluid velocity at the midchannel and the pressure gradient $\partial p / \partial x$ along the $x$-axis, for even $n$, are found 
to be

$$
\begin{aligned}
u_{x}(y)= & \frac{\partial}{\partial y} \psi\left(\rho, \frac{\pi}{2}\right) \\
= & \frac{-2 M\left(1+\varepsilon(n+1) \rho^{n}(-1)^{k}\right)}{\pi\left(1+2 \varepsilon(n+1) \rho^{n}(-1)^{k}-\varepsilon^{2}(n+1)^{2} \rho^{2 n}\right)} \\
& \times\left\{\frac{2\left(1+\rho^{2}\right)}{\left(1-\rho^{2}\right)^{2}+4 \rho^{2}}+\frac{1}{1-\varepsilon^{2}(n+1)^{2}}\right. \\
& \times\left[\frac { 1 } { 2 } \sum _ { m = 1 } ^ { n - 1 } \left[\Omega_{m}\left((m+2) \rho^{m+1}-m \rho^{m-1}\right)\right.\right. \\
& +\frac{\left(1-3 \rho^{2}\right)-\varepsilon^{2}(n+1)\left(1-(2 n+3) \rho^{2 n+2}\right)}{1+\rho^{2}} \\
& \left.\left.\left.\quad+\frac{4 \varepsilon n(-1)^{k} \rho^{n+2}\left(1-\rho^{4}\right)}{\left(1+\rho^{2}\right)^{3}}\right] \rho^{m-1}\right)\right] \sin \frac{m \pi}{2} \\
& \quad-\frac{2 \rho^{2}\left[\left(1-\rho^{2}\right)-\varepsilon^{2}(n+1)\left(1-\rho^{2 n+2}\right)\right]}{\left(1+\rho^{2}\right)^{2}} \\
& \left.\left.\quad+\frac{\varepsilon n \rho^{n}(-1)^{k}\left(\left(1-5 \rho^{4}\right)+n\left(1-\rho^{4}\right)\right)}{\left(1+\rho^{2}\right)^{2}}\right]\right\},
\end{aligned}
$$

where $n=2 k$ for $k \in \mathbf{N}$. Finally, the pressure gradient in the $x$-direction along the $y$-axis, for the same cases of $n \in \mathbf{N}$, is

$$
\frac{\partial p}{\partial x}=\left\{\frac{1+\varepsilon(n+1) \rho^{n} \cos ((n+1) \phi)}{1+2 \varepsilon(n+1) \rho^{n}+\varepsilon^{2}(n+1)^{2} \rho^{2 n}}\right\} H(\rho, n),
$$

where $0 \leq \rho \leq 1, \phi=0$ or $\pi$ and

$$
\begin{aligned}
& H(\rho, n)=\frac{4 M}{\pi\left(1-\varepsilon^{2}(n+1)^{2}\right)} \\
& \quad \times\left\{\sum _ { m = 1 } ^ { n - 1 } m \left[(m+1) \Omega_{m} \rho^{m-1}\right.\right. \\
& \quad+\frac{1}{\left(1-\rho^{2}\right)^{3}}\left[-4\left(1-\varepsilon^{2}(n+1)^{2}\right)\left(1+3 \rho^{2}\right) 2 \varepsilon^{2}(n+1)^{2} n \rho^{2 n}\left(1-\rho^{4}\right)\right) \\
& \quad+2 \varepsilon(n+1) \rho^{n}\left(1-\rho^{2}\right) \\
& \left.\left.\quad \times\left((n+1)(n+2)-(n-1)\left(n \rho^{2}-2\right)\right) \cos ((n+1) \phi)\right]\right\} .
\end{aligned}
$$


As a check, set $\varepsilon=0$; then the full solution, (3.10), reduces to the solution of the circle problem (see [9])

$$
\psi=\frac{2 M}{\pi}\left[\tan ^{-1}\left(\frac{2 \rho \sin \phi}{1-\rho^{2}}\right)+\left[\frac{\left(1-\rho^{2}\right)\left(1+\rho^{2}\right) \rho \sin \phi}{1-2 \rho^{2} \cos 2 \phi+\rho^{4}}\right]\right]
$$

Whereas, (3.12) reduces to

$$
\omega(1, \phi ; 0)=\frac{-4 M}{\pi}\left(\frac{1}{\sin \phi}\right)<0 \quad \forall 0<\phi<\pi,
$$

which predicts no separation of streamlines near the boundary (a known result from Rayleigh's problem [9]). The velocity profile at mid-channel becomes

$$
u_{x}(y)=\frac{-2 M}{\pi}\left\{\frac{2\left(1+y^{2}\right)}{\left(1-y^{2}\right)^{2}+4 y^{2}}+\frac{1-3 y^{2}}{1+y^{2}}-\frac{2 y^{2}\left(1-y^{2}\right)}{\left(1+y^{2}\right)^{2}}\right\} .
$$

For flow between two planes extended to infinity, i.e., two-dimensional Poiseuille flow for a channel, the known result is

$$
u_{x}(y)=-\frac{3 M}{2}\left(y^{2}-1\right), \quad p_{x}=-3 M
$$

$\S 4$. Odd $n$; convex source and concave sink $\left(\varepsilon \in I_{1}\right)$. It was observed that for all $n \geq 1, \varepsilon>1 /(n+1)^{2}$, vortices near the source appeared (see Fig. 1). For $n=1$ this has already been observed for a similar problem found in [10]. These vortices extend near the source as $\varepsilon \rightarrow(n+1)^{-1}$, eventually reaching it in the limit. Note that on the boundary mid-way between the source and sink, for $n \geq 5$, there appear regions of very low fluid velocity, or "stagnation." They occur for moderate to large values of $\varepsilon$ where $1 /(n+1)^{2}<\varepsilon<1 /(n+1)$.

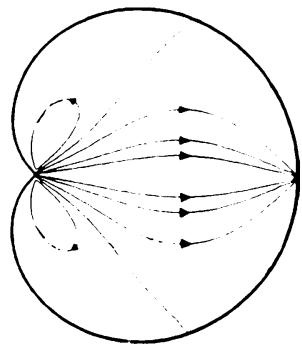

$\epsilon=0.45$

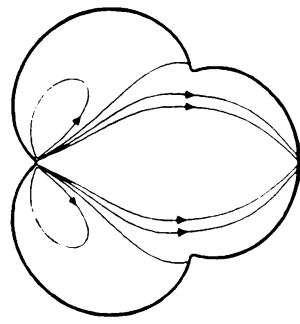

$\epsilon=0.21$

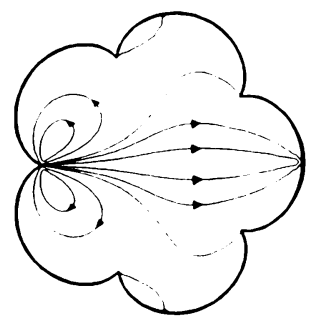

$\epsilon=0.16$

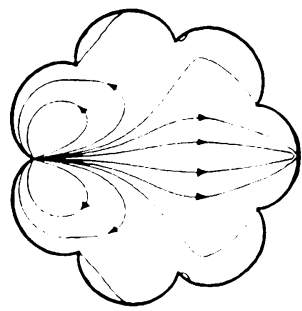

$\epsilon=0.122$

FIG. 1. Some solutions for $n=1,3,5,7$. 
§5. Even $n$; convex source and sink $\left(\varepsilon \in I_{1}\right)$. For small $n, \varepsilon>0$, no separation of flow occurs (Fig. 2). For the case $n=2$ this is expected (see [6]). For $n \geq 4$ regions of stagnant flow appear. The common feature of these geometries is that fluid enters and escapes the region from convex walls.

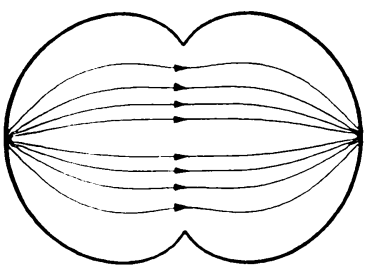

$\epsilon=0.31$

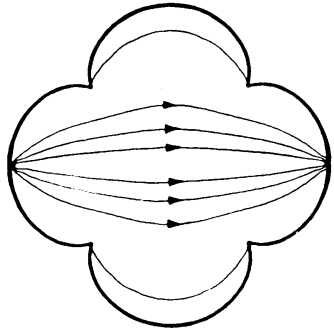

$\epsilon=0.19$

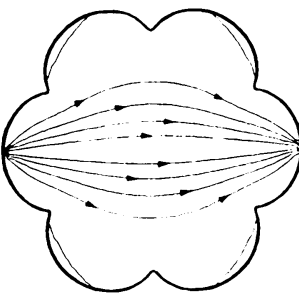

$\epsilon=0.12$

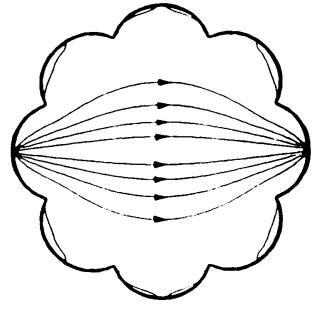

$\epsilon=0.09$

FIG. 2. Some solutions for $\varepsilon>0$ and $n=2,4,6,8$.

§6. Even $n$; concave source and sink $\left(\varepsilon \in I_{2}\right)$.

(i) General discussion. As in the odd $n$ case there are always vortices for $\varepsilon<$ $-1 /(n+1)^{2}$, but a new phenomenon occurs (Fig. 3). There is always a sufficient $\varepsilon$, in the range $\left(-(n+1)^{-1},-(n+1)^{-2}\right)$ for which an eddy from the source and an eddy from the sink meet to form one large free eddy, with two vortices interior (separatrix). A jet of fluid is formed running from the source to sink which occupies a narrow region and outside of which all fluid stays inside the pipe. This is a large class of boundary value problems containing a flow divider (see [10]). It is instructive to consider the case $n=4$. This is a model for a stenotic channel with concave source and sink. The flow is much different than the stenotic channel with convex source and sink though, since vortices appear in this case.

(ii) Effect of stenosis. To see the formation of the vortices, consider the diagrams in Fig. 4, for $n=4$. One can also follow the transition by considering the boundary vorticity (see Fig. 5). Note that at the limiting value $\varepsilon=-(4+1)^{-2}=-0.04$, the sign of the vorticity changes abruptly at $\phi=0, \pi$. It was observed that for the same curvature of source and sink the channel with stenosis (i.e., concave boundary points at $\phi=\frac{\pi}{2}, \frac{3 \pi}{2}$ ) experienced the formation of a free eddy later as $n$ gets larger. Hence this geometry hinders the formation of large vortices as $n$ increases. This
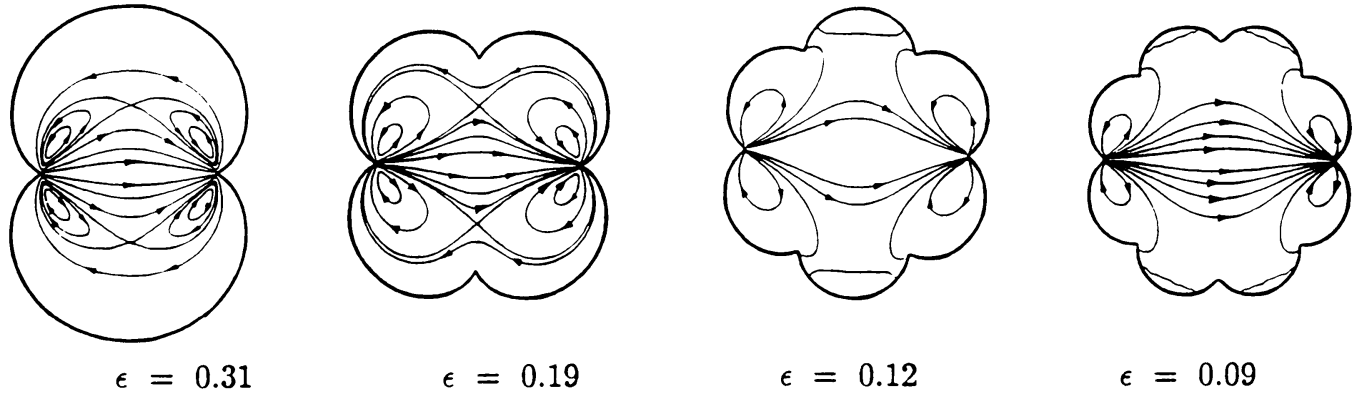

$$
\epsilon=0.19
$$

$$
\epsilon=0.12
$$$$
\epsilon=0.09
$$

Fig. 3. Some solutions for $\varepsilon<0$ and $n=2,4,6,8$. 


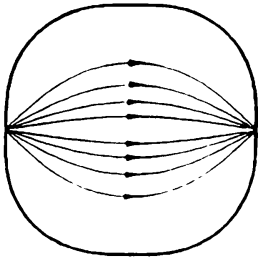

$\epsilon=-0.04$

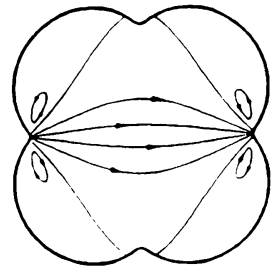

$\epsilon=-0.14$

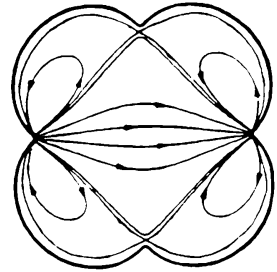

$\epsilon=-0.16$

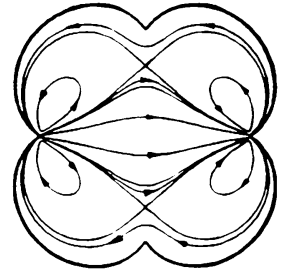

$\epsilon=-0.18$

Fig. 4. Transition to separatrices in $n=4$ case.

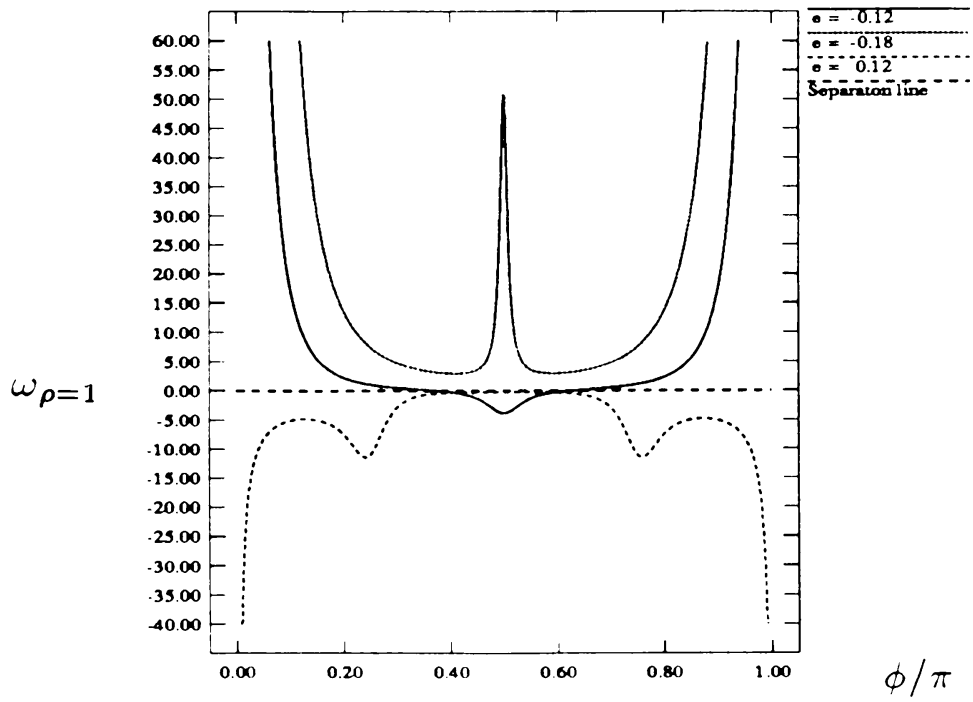

FIG. 5. Variation of boundary vorticity for $n=4$.

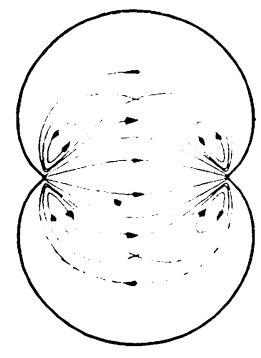

$\epsilon=0.2909$

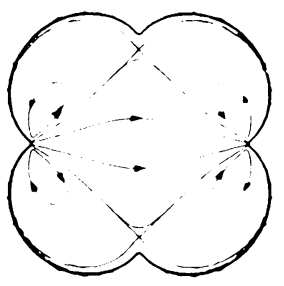

$\epsilon=0.1647$

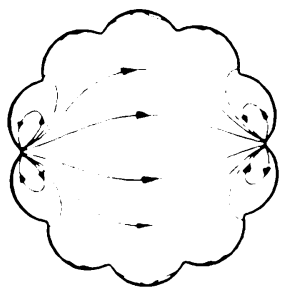

$\epsilon=0.0667$

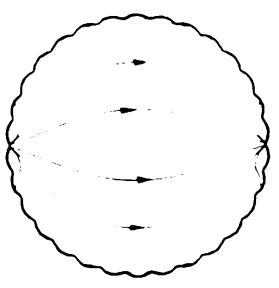

$\epsilon=0.0189$

FIG. 6. Some solutions with source curvature $\kappa=100$, for $n=$ $2,4,10,30$.

phenomenon also occurs for large $n$. A comparison of four different $n$ values is given in Fig. 6 where the curvature of the concave source and sink is $\kappa=100$.

$\S 7$. Large $n$. For large $n$ the boundary approaches the shape of a circle. For $|\varepsilon| \sim(n+1)^{-1}$ there is always some "roughness" of the surface. This has an effect on the fluid flow, as the boundary vorticity plots Fig. 8(a-c) indicate. 


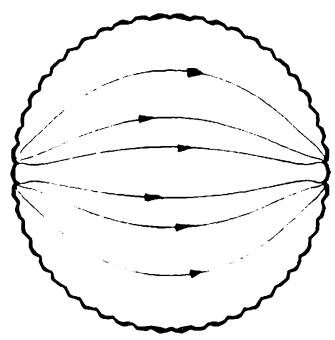

$\epsilon=0.0196$

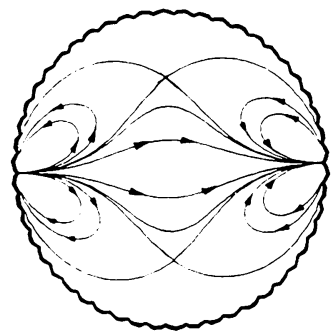

$\epsilon=-0.01945$

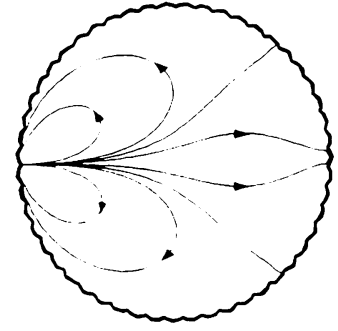

$\epsilon=0.0192$

FIG. 7. Some solutions for $n=50,50,51$.

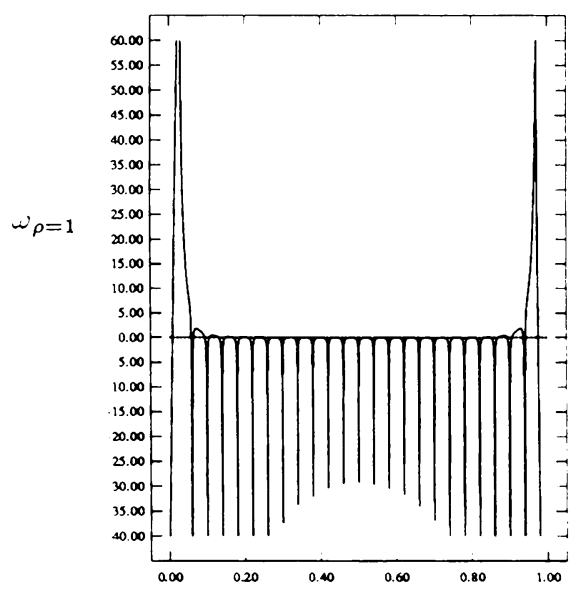

(a) $n=50 . \epsilon \geq 0$.

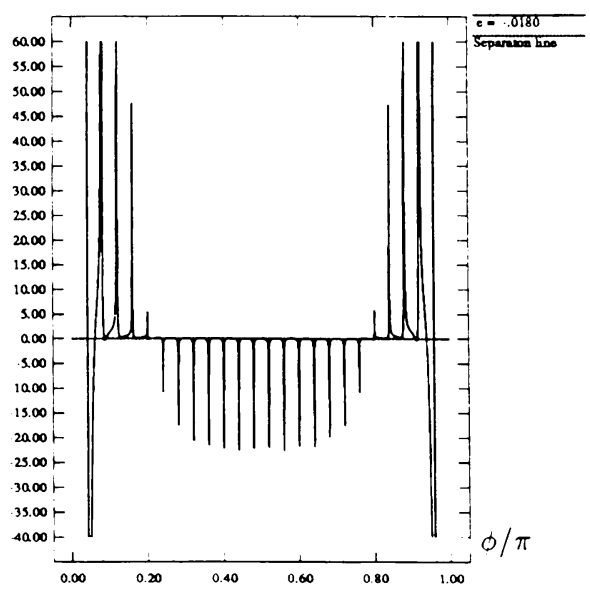

(b) $n=50, \epsilon \leq 0$

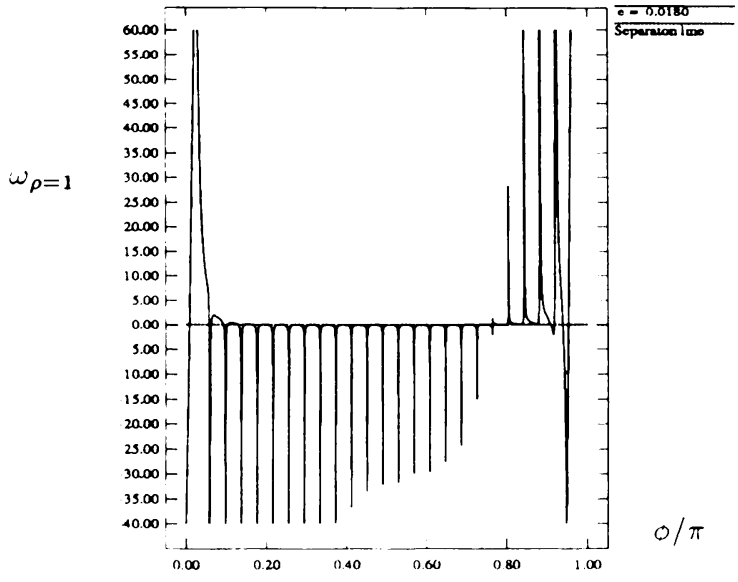

(c) $n=51, \epsilon \geq 0$.

FIG. 8. Variation of boundary vorticity for some cases of $n$. 
It is clear though that the most important aspect affecting the pattern of the fluid flow is the shape of the source and sink (see Figs. 1, 2, 3, and 7).

§8. Approximate model of flow in a rough channel. An approximate model for pipe flow is found by considering $n$ large and even, with $\varepsilon>0$. Then the region $x \in(-\delta, \delta)$ for $\varepsilon \ll \delta \ll 1$ simulates the creeping flow in a corrugated channel (Fig. 9).

The mid-channel velocity profile of the two dimensional Poiseuille flow for a channel is compared to that of the circle problem and the rough channel $(n=20$ with smooth boundary at $\phi=\pi / 2$, and $n=22$ with cusp on boundary at $\phi=\pi / 2$ ) in Fig. 10. Both the circular and corrugated boundaries give rise to an increase in
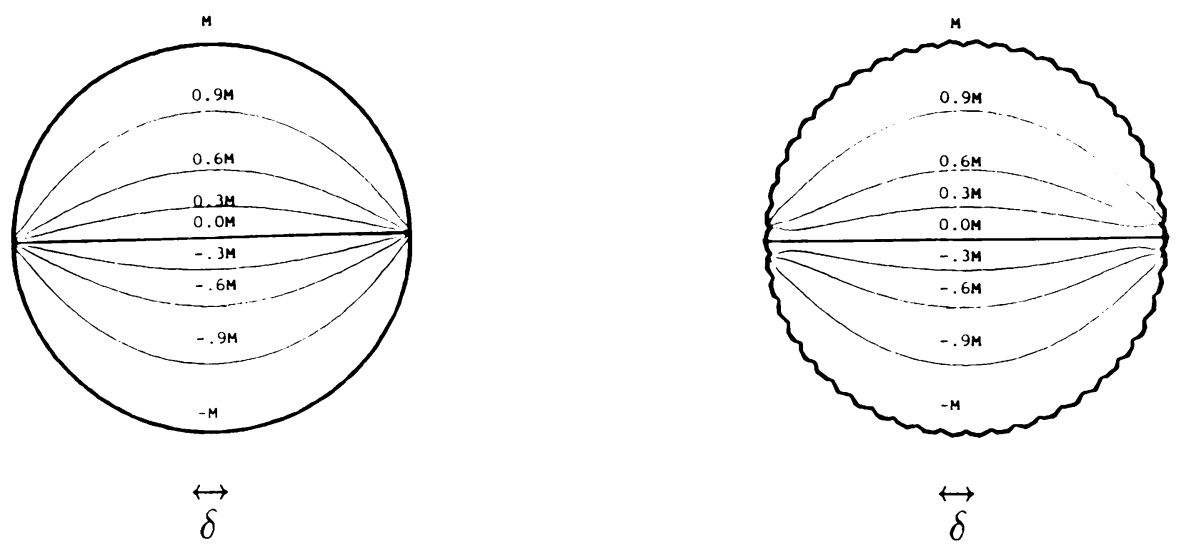

FIG. 9. Comparison of circle streamlines with case $n=50, \varepsilon=$ 0.0196 .

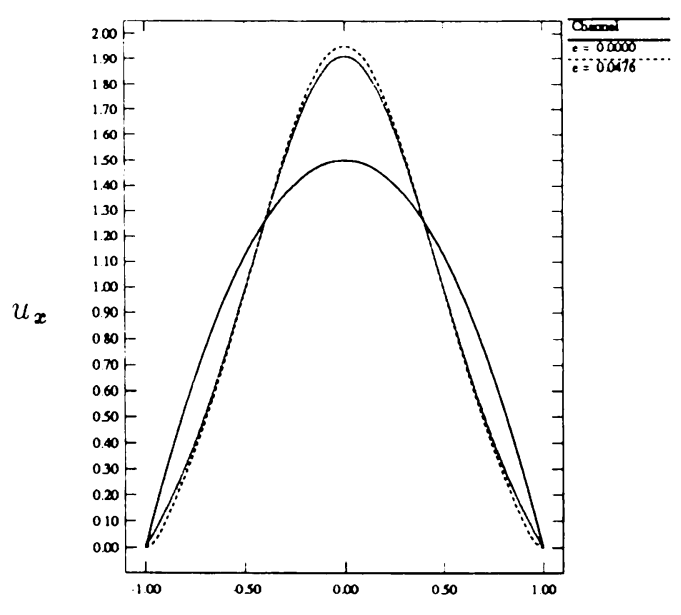

$y$-axis (normalized)

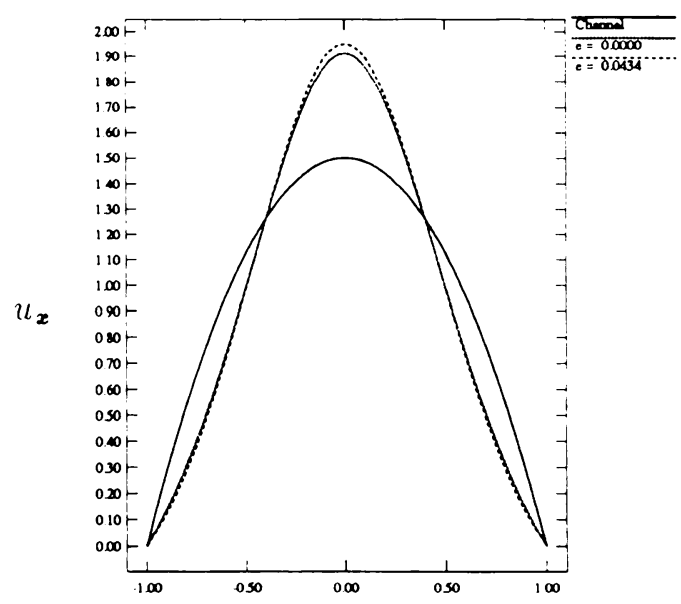

$y$-axis (normalized)

$$
n=20 \quad n=22
$$

FIG. 10. Mid-channel velocity ( $x$-component of velocity on the line $x=0.0)$. 
mid-channel velocity at $y=0$, but the presence of corrugations on the boundary gives the highest mid-channel velocity at $y=0$. However, a deviation of the mid-channel velocity at the boundary (see Fig. 11) for the circle problem and rough channel only occurs inside a cusp. The result is qualitatively similar to that found in Fig. 2 of [5]. Similar comparisons are also made for their pressure gradients in the $x$-direction along the $x$-axis in Fig. 12. On the contrary, the pressure gradient is decreased for

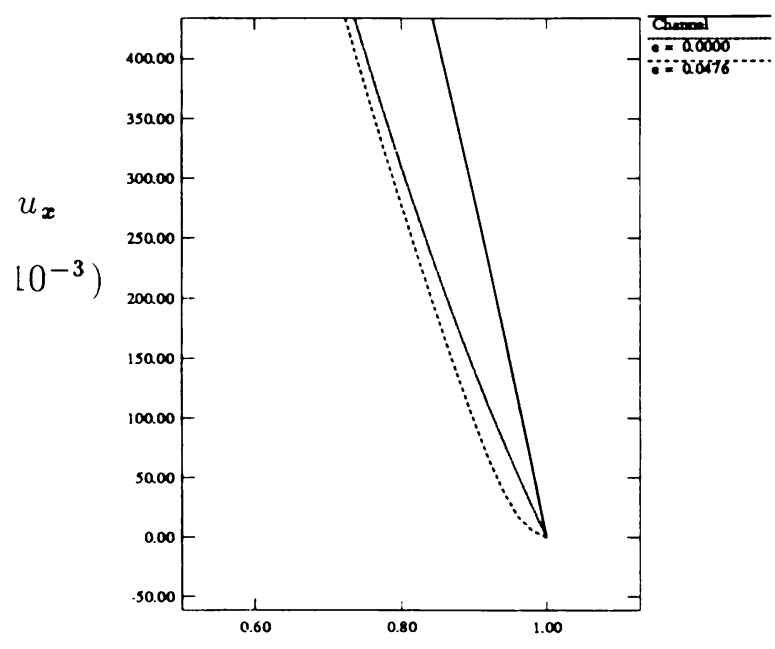

$y$-axis (normalized)

$$
n=20
$$

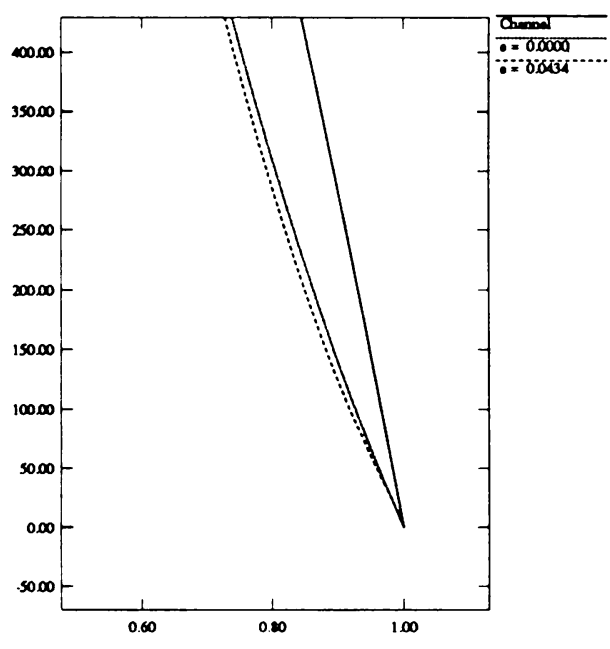

$y$-axis (normalized)

$n=22$

FIG. 11. Velocity near the boundary on the line $x=0.0$.

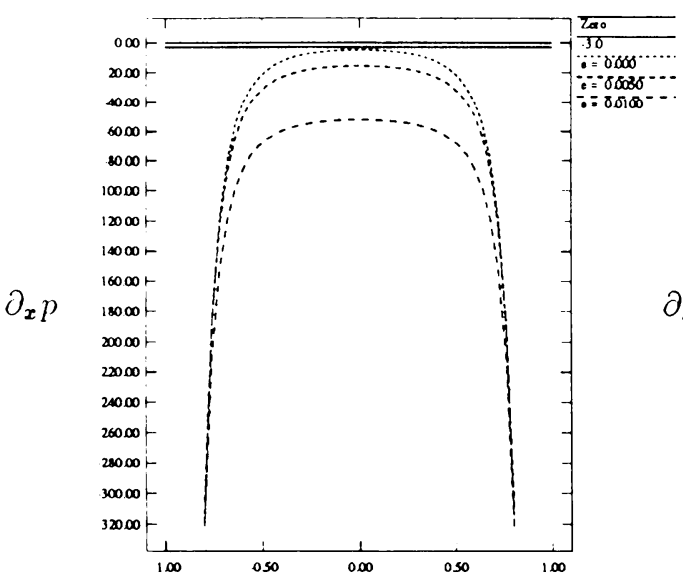

$x$-axis

$n=20$

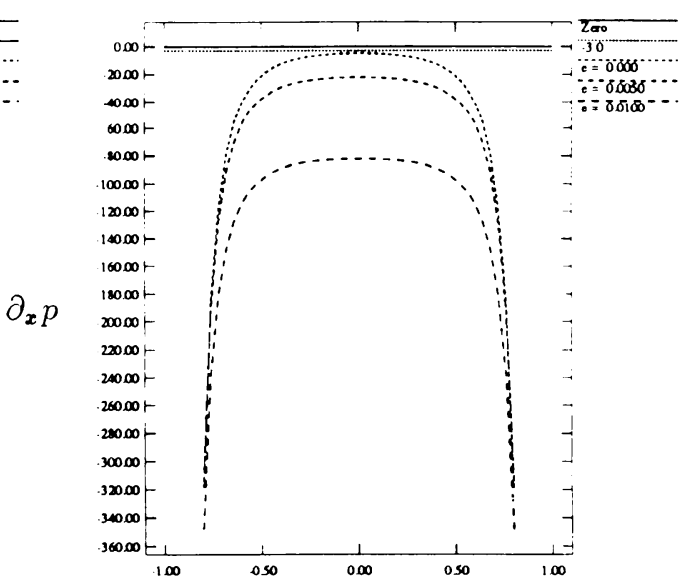

$x$-axis

$n=22$

FIG. 12. Pressure gradient in $x$-direction on the line $y=0.0$. 
the circle and corrugated boundaries at $y=0, x \in(-\delta, \delta)$, as $\varepsilon$ increases. Also, the presence of corrugations gives the lowest pressure gradient at mid-channel. This result is compatible with that found for the mid-channel velocity. So it is conjectured that for the same amount of flux the mid-channel velocity is increased (or the pressure gradient at mid-channel is decreased) with the presence of corrugations.

Acknowledgments. The authors wish to thank Professor K. B. Ranger for suggesting the problem treated in this article, and for providing many helpful discussions, which made this paper possible.

Appendix. Recall from (3.7c) that

$$
\begin{gathered}
B_{m}+\varepsilon\left[(n+1) B_{n+m}-(n+1-m) B_{n-m}\right]=\frac{M}{\pi}\left((-1)^{m}-1\right), \quad 1 \leq m \leq n-1, \\
B_{m}+\varepsilon(n+1) B_{m+n}=\frac{M}{\pi}\left((-1)^{m}-1\right), \quad n \leq m .
\end{gathered}
$$

For $m \geq n$ set $B_{m}=A(-1)^{m}+B$ to be the form of the solution of (A.1) with a suitable choice of constants $A$ and $B$. Note that neither $A(-1)^{m}$ nor $B$ alone will yield solutions to the homogeneous difference equations if $|\varepsilon|<1 /(n+1)$. (A.1) implies

$$
\begin{aligned}
& A(-1)^{m}+B+\varepsilon(n+1)\left[A(-1)^{m+n}+B\right]=\frac{M}{\pi}\left((-1)^{m}-1\right), \quad m \geq n, \\
& \Rightarrow \quad\left(1+(-1)^{n} \varepsilon(n+1)\right) A+(1+\varepsilon(n+1)) B=\frac{M}{\pi}\left((-1)^{m}-1\right), \quad m \geq n, \\
& \Rightarrow \quad A=\frac{M}{\pi}\left[\frac{1}{1+(-1)^{n} \varepsilon(n+1)}\right], \quad B=-\frac{M}{\pi}\left[\frac{1}{1+\varepsilon(n+1)}\right] .
\end{aligned}
$$

Then, for $m>n$

$$
\begin{aligned}
B_{m} & =\frac{M}{\pi}\left[\frac{(-1)^{m}}{1+(-1)^{n} \varepsilon(n+1)}-\frac{1}{1+\varepsilon(n+1)}\right] \\
& =\frac{M}{\pi}\left[\frac{(-1)^{m}\left(1-(-1)^{n} \varepsilon(n+1)\right)}{1-\varepsilon^{2}(n+1)^{2}}-\frac{1-\varepsilon(n+1)}{1-\varepsilon^{2}(n+1)^{2}}\right] \\
& =\frac{M}{\pi}\left[\frac{\left((-1)^{m}-1\right)-\varepsilon(n+1)\left((-1)^{m+n}-1\right)}{1-\varepsilon^{2}(n+1)^{2}}\right] .
\end{aligned}
$$

Due to the form of (A.2) and the solutions of $B_{m}$ 's when $\varepsilon=0$ (i.e., $B_{m}=$ $\left.\frac{M}{\pi}\left((-1)^{m}-1\right)\right)$, set

$$
B_{m}=\frac{M}{\pi}\left[\frac{\left((-1)^{m}-1\right)+\Omega_{m}}{1-\varepsilon^{2}(n+1)^{2}}\right], \quad 1 \leq m \leq n-1
$$


Substituting this and (A.2) into (A.1) gives the following equation, which holds for $1 \leq m \leq n-1$ :

$$
\begin{aligned}
& \frac{M}{\pi}\left[\frac{1}{1-\varepsilon^{2}(n+1)^{2}}\right]\left\{\left((-1)^{m}-1\right)+\Omega_{m}\right. \\
& +\varepsilon\left(\varepsilon(n+1)\left(\left((-1)^{m+n}-1\right)-\varepsilon(n+1)\left((-1)^{m}-1\right)\right)\right. \\
& \left.\left.-(n+1-m)\left((-1)^{m+n}-1+\Omega_{n-m}\right)\right)\right\} \\
& =\frac{M}{\pi}\left((-1)^{m}-1\right),
\end{aligned}
$$

and this reduces to

$$
\Omega_{m}-\varepsilon(n+1-m) \Omega_{n-m}=\varepsilon m\left(1-(-1)^{m+n}\right),
$$

which is true for $1 \leq m \leq n-1$. (A.3) can be solved by trying out several $n$ 's, and using mathematical induction (for $n$ ) to verify the general result. The result is found to be

$$
\Omega_{m}=-\varepsilon\left\{\frac{\left((-1)^{m+n}-1\right) m+\varepsilon\left((-1)^{m}-1\right)(n-m)(n+1-m)}{1-\varepsilon^{2}(m+1)(n+1-m)}\right\},
$$

for $1 \leq m \leq n-1$.

\section{REFERENCES}

[1] A. C. Payatakes, Chi Tien, and R. M. Turian, A new model for granular porous media, A. I. Ch. E. J. 11, 58-76 (1973)

[2] N. Phan-Thien, C. J. Goh, and M. B. Bush, Viscous flow through a corrugated tube by boundary element method, Z. Angew. Math. Phys. 36, 475-480 (1985)

[3] H. Tennekes and J. L. Lumley, A First Course in Turbulence, M.I.T. Press, Cambridge, MA, 1972

[4] N. Phan-Thien, On the Stokes flow of viscous fluids through corrugated pipes, A. S. M. E. J. Appl. Mech. 47, 961-963 (1980)

[5] M. Lessen and P. S. Huang, Poiseuille flow in a pipe with axially symmetric wavey walls, Physics Fluids 19, 945-950 (1976)

[6] A. M. J. Davis and K. B. Ranger, A model for Stokes flow through a stenotic channel, Phys. Fluids A 1, 193-198

[7] D. E. R. Godfrey, Theoretical Elasticity and Plastics for Engineers, Thames and Hudson, London, 1959 , pp. 58-59

[8] E. G. Philips, Functions of a Complex Variable with Applications, Oliver and Boyd, Interscience Publishers Inc., 1958, p. 31

[9] Lord Rayleigh, Scientific Papers, Vol. IV, Cambridge, 1900

[10] K. B. Ranger and H. Brenner, A model for Stokes flow in a two-dimensional bifurcating channel, J. Fluid Mech. 152, 1-13 (1985) 